\title{
RESEARCH REPOQRTS
}

\section{Institutional Experience with Voriconazole Compared with Liposomal Amphotericin B as Empiric Therapy for Febrile Neutropenia}

Nadine Shehab, Pharm.D., Daryl D. DePestel, Pharm.D., Emily R. Mackler, Pharm.D., Curtis D. Collins, Pharm.D., M.S., Kathleen Welch, M.S., M.P.H., and Harry P. Erba, M.D., Ph.D.

Study Objective. To assess the effectiveness, safety, and cost of empiric treatment of febrile neutropenia before and after implementing an algorithm in which voriconazole was substituted for liposomal amphotericin B (L-AmB).

Design. Retrospective cohort analysis.

Setting. An 850-bed tertiary care hospital, which is also a referral site for patients with acute leukemia.

Patients. Fifty-five adult patients who started empiric antifungal therapy for febrile neutropenia between January 1, 2002, and December 31, 2003, encompassing 58 treatment episodes (defined as a hospitalization during which empiric antifungal therapy was administered).

Measurements and Main Results. Medical charts, including patients' pharmacy and laboratory data, were reviewed. Twenty-six and 32 episodes of L-AmB and voriconazole use, respectively, were identified. No significant differences between the L-AmB and voriconazole groups were noted at baseline. Rates of fever resolution ( $54 \%$ vs $59 \%, \mathrm{p}=0.791$ ) and breakthrough invasive fungal infections ( $11 \%$ vs $12 \%$, p >0.999) were similar for the L-AmB and voriconazole episodes. Premature drug discontinuation due to the prescriber's perceived lack of efficacy occurred most frequently in the voriconazole group ( $25 \%$ vs $8 \%, \mathrm{p}=0.160)$. Survival was significantly higher in the voriconazole than in the L-AmB group ( $100 \%$ vs $77 \%, p=0.006)$. Adverse effects that were significantly more common in the L-AmB group than in the voriconazole group were elevated serum creatinine levels ( $27 \%$ vs $3 \%, \mathrm{p}=0.017)$ and electrolyte disturbances ( $19 \%$ vs $0 \%, \mathrm{p}=0.014$ ). Adverse effects reported more frequently in the voriconazole group than in the L-AmB group were visual disturbances ( $9 \%$ vs $0 \%, \mathrm{p}=0.245$ ) and elevated hepatic enzyme levels (9\% vs $8 \%, \mathrm{p}>0.999$ ). Mean drug expenditures/episode for initial empiric antifungal therapy were lower for voriconazole than for L-AmB (\$1593 vs $\$ 4144$, or $\$ 153$ vs \$380/day).

Conclusion. Our institution's algorithm incorporating voriconazole into the empiric management of febrile neutropenia was associated with effectiveness outcomes comparable to those observed with L-AmB as well as a lower frequency of adverse effects and overall expenditures for antifungal drugs.

Key Words: voriconazole, liposomal amphotericin B, L-AmB, febrile neutropenia, antifungals, hematology, empiric therapy.

(Pharmacotherapy 2007;27(7):970-979) 
Empiric antifungal therapy with amphotericin B deoxycholate is considered the standard of care for patients with febrile neutropenia $(<500$ polymorphonuclear neutrophils $/ \mathrm{mm}^{3}$ ) that persists despite broad-spectrum antibacterial therapy. ${ }^{1}$ Liposomal amphotericin B (L-AmB) has similar effectiveness in patients with febrile neutropenia and is generally better tolerated than amphotericin B deoxycholate. However, it is associated with a substantially higher acquisition cost. ${ }^{2,3}$ Anecdotal evidence suggests that voriconazole, a second-generation azole antifungal agent, is increasingly being used in lieu of L-AmB as empiric therapy for febrile neutropenia despite a lack of data to suggest its equivalency to amphotericin products. ${ }^{4}$ Voriconazole is active against most fungi that contribute to invasive fungal infections observed in immunocompromised patients, including those due to relatively uncommon but increasingly important emerging pathogens such as Scedosporium and Fusarium species. ${ }^{5-9}$ Other potential benefits of voriconazole compared with L-AmB are its availability in oral formulations, its favorable safety profile, and its low acquisition cost. ${ }^{4,10-12}$

Our institution developed an algorithm in which voriconazole is the preferred agent for adult patients who underwent chemotherapy for hematologic cancer and experienced febrile neutropenia (Figure 1). Thus, our primary objective was to assess the impact of substituting voriconazole for L-AmB on effectiveness, safety, and cost in this population. Our secondary objective was to characterize how empiric anti-fungal therapy was being used before (January 1-December 31, 2002) and after (January 1-December 31, 2003) implementation of the algorithm.

\section{Methods}

\section{Study Design, Setting, and Patients}

This retrospective cohort study was based on a

From the Department of Clinical Sciences, College of Pharmacy (Drs. Shehab and DePestel), and the Department of Internal Medicine, School of Medicine (Dr. Erba), University of Michigan; and the Department of Pharmacy Services (Drs. Shehab, DePestel, Mackler, and Collins), Center for Statistical Consultation and Research (Ms. Welch), and the Department of Internal Medicine, Division of Hematology-Oncology (Dr. Erba), University of Michigan Health System, Ann Arbor, Michigan.

Presented at the annual meeting of the American College of Clinical Pharmacy, San Francisco, California, October 23-26, 2005.

Address reprint requests to Daryl D. DePestel, Pharm.D., University Hospital, B2D301, 1500 East Medical Center Drive, Ann Arbor, MI 48109-0008; e-mail: daryldd@ umich.edu. review of medical charts including pharmacy and laboratory data. It was conducted at the University of Michigan Hospital, which is an 850-bed tertiary care center. The hospital is also a large referral site for patients with acute leukemia, with more than 70 such patients admitted annually. The institutional review board of the University of Michigan approved this study; informed consent was not required for the study due to its retrospective nature.

Patients were eligible if they were at least 18 years old; if they received chemotherapy to treat leukemia, lymphoma, or another hematologic cancer; if they had a documented diagnosis of febrile neutropenia; and if they received empiric antifungal therapy with L-AmB between January 1 and December 31, 2002 or with voriconazole between January 1 and December 31, 2003. Patients were excluded if they had a documented invasive fungal infection at admission or within 24 hours of first receiving empiric antifungal therapy. ${ }^{13}$ Patients who had undergone or were undergoing hematopoietic stem cell transplantation were also excluded.

\section{Data Collection}

To compare the two treatments, L-AmB versus voriconazole, we collected data according to episode. A treatment episode was defined as a single hospital admission during which a patient received at least one dose of $\mathrm{L}-\mathrm{AmB}$ or voriconazole as the initial empiric antifungal agent; these

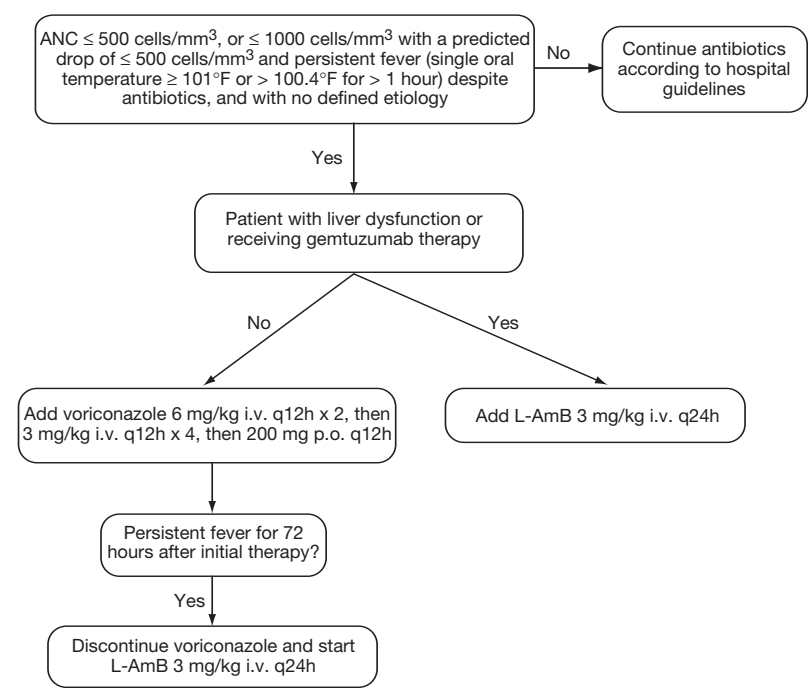

Figure 1. University of Michigan Hospital febrile neutropenia treatment algorithm. $\mathrm{ANC}=$ absolute neutrophil count; L-AmB = liposomal amphotericin B. 
episodes were assigned to the L-AmB or voriconazole group accordingly.

We recorded the patients' demographic characteristics, primary diagnosis, and chemotherapy regimens. Receipt of systemic antifungal prophylaxis, granulocyte colony-stimulating factor (filgrastim), and/or granulocyte-macrophage colony-stimulating factor (sargramostim), as well as the duration of neutropenia (absolute neutrophil count $<500$ cells $/ \mathrm{mm}^{3}$ ) and fever (temperature $\geq$ $100.4^{\circ} \mathrm{F}\left[\geq 38^{\circ} \mathrm{C}\right]$ ) were noted. Any modifications to antibacterial regimens within 48 hours before the start of empiric antifungal therapy were also documented.

We also recorded the use of antifungal drugs, including their dosages, durations of therapy, routes of administration, and any premedication with acetaminophen, meperidine, or diphenhydramine. The number and fungal etiology (if available) of all breakthrough invasive fungal infections were documented. Breakthrough invasive fungal infections were further classified as proven, probable, or possible on the basis of the Mycoses Study Group criteria for disease certainty. ${ }^{13}$ A hematologist-oncologist reviewed and confirmed these classifications. Utilization data were collected for any antifungal agents that were started if therapy with L-AmB or voriconazole was discontinued or if an antifungal agent was added to L-AmB or voriconazole therapy.

\section{Effectiveness Outcomes}

Measures of effectiveness were the resolution of fever (temperature $<100.4^{\circ} \mathrm{F}\left[<38^{\circ} \mathrm{C}\right]$ for at least $24 \mathrm{hrs}$ ) during the period of neutropenia, no discontinuation of an antifungal agent because of the prescriber's perceived lack of efficacy before the patient recovered from neutropenia, absence of a breakthrough invasive fungal infection within 7 days after the end of therapy, and survival within 7 days after the end of therapy.

\section{Safety Outcomes}

We documented the following adverse laboratory effects that occurred during empiric antifungal therapy: alkaline phosphatase, aspartate aminotransferase, alanine aminotransferase, and bilirubin levels that were 5 times baseline in patients whose baseline values were less than 2 times the upper limit of normal (we noted increases of 3 times baseline when patient's baseline values were 2-5 times the upper limit of normal); and instances of hypokalemia (potassium concentration $\leq 3.0 \mathrm{mEq} / \mathrm{L}$ ), hypomagnesemia (magnesium concentration $\leq 1.5$ $\mathrm{mg} / \mathrm{dl}$ ), and nephrotoxicity (serum creatinine levels $>1.5$ times the patient's baseline value).

We also documented infusion-related reactions, such as chills, fever, or a rash that developed as a direct result of the empiric antifungal agent, as well as the need for dialysis during empiric antifungal therapy. We recorded other adverse reactions, including visual disturbances, that occurred during treatment and that were documented in the medical record. Finally, we reported any treatment discontinuation due to a documented adverse effect or a concern about an effect.

\section{Composite Outcome}

The composite outcome consisted of resolution of fever during the period of neutropenia, absence of a breakthrough invasive fungal infection within 7 days after the end of therapy, no discontinuation of antifungal therapy secondary to efficacy or safety concerns, and survival within 7 days after the end of therapy.

\section{Health Care Resource Utilization}

Health care resource utilization was analyzed by assessing data obtained from the University of Michigan Hospitals and Health Centers data warehouse. Mean hospital length of stay, antifungal drug costs, total pharmacy costs, and total hospital costs were compared between the $\mathrm{L}-\mathrm{AmB}$ and voriconazole groups. Analyses were limited to costs from the time empiric antifungal therapy was started until all antifungal drugs were discontinued. Costs associated with antifungal drugs that were added to L-AmB or voriconazole therapy or with drugs begun after the discontinuation of $\mathrm{L}-\mathrm{AmB}$ or voriconazole were also collected.

\section{Statistical Analysis}

The Student $t$ test was used for all comparisons of continuous data between the groups. When normality could not be assumed, the MannWhitney $U$ test was applied. The $\chi^{2}$ or Fisher exact test was used for all other comparisons between voriconazole and L-AmB. An $\alpha$ of 0.05 was applied. Results are reported as the number (percentage) of episodes, the mean $\pm \mathrm{SD}$, or the mean dollars. Statistical analyses were performed using SAS, version 9.1 (SAS Institute Inc., Cary, NC). 
Table 1. Demographic and Clinical Characteristics of the Patients During the 58 Episodes

\begin{tabular}{|c|c|c|c|}
\hline Characteristic & $\begin{array}{l}\text { L-AmB Group } \\
\text { (26 episodes) }\end{array}$ & $\begin{array}{l}\text { Voriconazole Group } \\
\text { (32 episodes) }\end{array}$ & $\mathrm{p}$ Value \\
\hline \multicolumn{4}{|c|}{ No. (\%) of Episodes } \\
\hline Sex & & & 0.596 \\
\hline Male & $14(54)$ & $20(63)$ & \\
\hline Female & $12(46)$ & $12(38)$ & \\
\hline Race & & & $>0.999$ \\
\hline Caucasian & $25(96)$ & $30(94)$ & \\
\hline African-American & $0(0)$ & $1(3)$ & \\
\hline Other & $1(4)$ & $1(3)$ & \\
\hline \multicolumn{4}{|l|}{ Primary diagnosis } \\
\hline New AML & $13(50)$ & $20(63)$ & 0.427 \\
\hline Relapsed or refractory AML & $9(35)$ & $4(13)$ & 0.061 \\
\hline Non-Hodgkin's lymphoma & $2(8)$ & $3(9)$ & $>0.999$ \\
\hline Multiple myeloma & $2(8)$ & $0(0)$ & 0.197 \\
\hline Other ${ }^{a}$ & $0(0)$ & $5(16)$ & 0.058 \\
\hline Systemic antifungal prophylaxis & $17(65)$ & $18(56)$ & 0.592 \\
\hline CSF therapy & $16(62)$ & $16(50)$ & 0.434 \\
\hline $\begin{array}{l}\text { Modification of antibacterial } \\
\text { therapy }^{\text {b }}\end{array}$ & $15(58)$ & $15(47)$ & 0.441 \\
\hline \multirow[t]{2}{*}{ Chemotherapy $^{\mathrm{c}}$} & $6(23)$ & $8(25)$ & $>0.999$ \\
\hline & \multicolumn{2}{|c|}{ Mean \pm SD } & \\
\hline Age (yrs) & $57.2 \pm 16.7$ & $48.6 \pm 17.2$ & 0.061 \\
\hline Body weight (kg) & $77.8 \pm 14.8$ & $82.5 \pm 17.1$ & 0.364 \\
\hline \multicolumn{4}{|l|}{ Duration (days) } \\
\hline Neutropenia & $10.6 \pm 5.4$ & $10.2 \pm 7.4$ & 0.331 \\
\hline Fever & $5.1 \pm 2.1$ & $5.8 \pm 3.6$ & 0.418 \\
\hline CSF therapy & $10.8 \pm 5.4$ & $11.4 \pm 6.2$ & 0.838 \\
\hline
\end{tabular}

L-AmB = liposomal amphotericin B; AML = acute myeloid leukemia; CSF = colony-stimulating factor. ${ }^{\mathrm{a}}$ One episode each of acute promyelocytic leukemia, acute lymphocytic leukemia, chronic myeloid leukemia, Hodgkin's disease, and myelodysplastic syndrome.

${ }^{\text {bUp to }} 48$ hours before the start of empiric antifungal therapy.

'Intermediate- or high-dose cytarabine for induction or reinduction.

\section{Results}

\section{Patients' Baseline Characteristics}

Fifty-five patients encompassing 58 treatment episodes met the inclusion criteria. Twenty-six (45\%) of the episodes represented L-AmB use in 2002, and 32 (55\%) represented voriconazole use in 2003.

Table 1 summarizes the patients' demographic and clinical characteristics. The two study groups were similar with respect to all baseline clinical characteristics. More episodes of refractory or relapsed acute myeloid leukemia were noted in the L-AmB group than in the voriconazole group; however, the difference was not significant (35\% vs $13 \%$; $=0.061$ ).

\section{Effectiveness}

Table 2 summarizes the effectiveness outcomes. No statistically significant differences were noted between the groups in terms of the number of episodes in which fever resolved during neutropenia or in which a breakthrough invasive fungal infection occurred. Three possible breakthrough invasive fungal infections occurred in the L-AmB group. In two, bronchoalveolar lavage fluid was positive for yeast. One proven and three possible breakthrough invasive fungal infections were observed in the voriconazole group. The proven infection was a fungal pneumonia due to Mucor species. In all other possible breakthrough episodes, tests of the bronchoalveolar lavage fluid yielded negative results.

Empiric antifungal agents were discontinued because of a perceived lack of efficacy more often with voriconazole than with L-AmB, but the difference was not statistically significant. In 20 (77\%) of 26 episodes involving L-AmB use, patients survived as long as 7 days after the end of therapy compared with all 32 (100\%) episodes in the voriconazole group $(\mathrm{p}=0.006)$. Multiple logistic regression analysis was performed to adjust for the high number of relapsed or refractory episodes of acute myeloid leukemia in 
Table 2. Effectiveness and Composite Outcomes

\begin{tabular}{|c|c|c|c|}
\hline Outcome & $\begin{array}{l}\text { L-AmB Group } \\
\text { (26 episodes) }\end{array}$ & $\begin{array}{l}\text { Voriconazole Group } \\
\text { (32 episodes) }\end{array}$ & $\mathrm{p}$ Value \\
\hline \multirow[t]{2}{*}{$\begin{array}{l}\text { Time to fever resolution, }{ }^{\text {a }} \\
\text { mean } \pm \text { SD (days) }\end{array}$} & $5.3 \pm 3.9$ & $4.9 \pm 3.6$ & 0.706 \\
\hline & \multicolumn{2}{|c|}{ No. (\%) of Episodes } & \\
\hline Resolution of fever ${ }^{\mathrm{a}}$ while neutropenic & $14(54)$ & $19(59)$ & 0.791 \\
\hline No breakthrough invasive fungal infection ${ }^{\mathrm{b}}$ & $23(88)$ & $28(88)$ & $>0.999$ \\
\hline No discontinuation due to prescriber's & & & \\
\hline $\begin{array}{l}\text { perceived lack of efficacy } \\
\text { Survival }^{\text {b }}\end{array}$ & $\begin{array}{l}24(92) \\
20(77)\end{array}$ & $\begin{array}{l}24(75) \\
32(100)\end{array}$ & $\begin{array}{l}0.160 \\
0.006\end{array}$ \\
\hline Composite & $7(27)$ & $13(41)$ & 0.405 \\
\hline
\end{tabular}

$\mathrm{L}-\mathrm{AmB}=$ liposomal amphotericin B.

${ }^{\mathrm{a}}$ Temperature $<100.4^{\circ} \mathrm{F}\left(<38^{\circ} \mathrm{C}\right)$ for at least 24 hours.

${ }^{b}$ Within 7 days after the end of therapy.

Table 3. Safety Outcomes

\begin{tabular}{|c|c|c|c|}
\hline \multirow[b]{2}{*}{ Outcome } & \multicolumn{2}{|c|}{ No. (\%) of Episodes } & \multirow[b]{2}{*}{$\mathrm{p}$ Value } \\
\hline & $\begin{array}{l}\text { L-AmB Group } \\
\text { (26 episodes) }\end{array}$ & $\begin{array}{l}\text { Voriconazole Group } \\
\text { (32 episodes) }\end{array}$ & \\
\hline Infusion-related reactions & $2(8)$ & $1(3)$ & 0.582 \\
\hline Nephrotoxicity ${ }^{\mathrm{a}}$ & $7(27)$ & $1(3)$ & 0.017 \\
\hline Dialysis & $2(8)$ & $0(0)$ & 0.197 \\
\hline Hypokalemia or hypomagnesemia & $5(19)$ & $0(0)$ & 0.014 \\
\hline Visual disturbances & $0(0)$ & $3(9)$ & 0.245 \\
\hline Altered liver function ${ }^{\mathrm{b}}$ & $2(8)$ & $3(9)$ & $>0.999$ \\
\hline Discontinuation of therapy ${ }^{c}$ & $1(4)$ & $4(13)$ & 0.367 \\
\hline
\end{tabular}

the L-AmB group. The difference in survival between the groups was still in the direction expected and remained significant $(\mathrm{p}=0.045)$.

\section{Safety}

\section{Infusion-Related Reactions}

Infusion-related reactions were reported in more L-AmB episodes than voriconazole episodes (Table 3). These reactions in the L-AmB group were one episode of dyspnea, flushing, laryngospasm, weakness, and dizziness and one episode of nausea. Premedication was administered before 24 (92\%) L-AmB infusions.

\section{Hepatotoxicity and Nephrotoxicity}

At baseline, L-AmB and voriconazole episodes were associated with similar rates of serum creatinine levels more than $2.0 \mathrm{mg} / \mathrm{dl}$ ( $4 \%$ vs $0 \%$, $\mathrm{p}=0.448$ ) or hepatic enzyme levels more than 3 times baseline (15\% vs 9\%, p=0.691). During empiric antifungal therapy, the groups did not significantly differ in the number of episodes in which hepatic enzyme levels were elevated. Hypokalemia, hypomagnesemia, and nephrotoxicity occurred significantly more frequently in L-AmB episodes than in voriconazole episodes.

Two patients in the L-AmB group required dialysis while they were receiving empiric antifungal therapy. In both episodes, therapy with LAmB was not the direct cause of dialysis; however, it contributed to worsening renal function.

\section{Visual Disturbances}

Three cases of visual disturbances were reported in the voriconazole group versus none in the L-AmB group; this difference was not statistically significant (Table 3 ). The visual disturbances were two episodes of visual hallucinations and one episode of blurred vision.

\section{Other Adverse Reactions}

Adverse reactions other than those previously described occurred in three (12\%) L-AmB 
Table 4. Treatment Durations and Costs

\begin{tabular}{|c|c|c|}
\hline Measure & $\begin{array}{l}\text { L-AmB Group } \\
\text { (26 episodes) }\end{array}$ & $\begin{array}{l}\text { Voriconazole Group } \\
\text { (32 episodes) }\end{array}$ \\
\hline & \multicolumn{2}{|c|}{ Mean \pm SD } \\
\hline \multicolumn{3}{|l|}{ Treatment duration (days) } \\
\hline Intravenous & $10.9 \pm 8.1$ & $5.5 \pm 4.1$ \\
\hline Oral & NA & $7.1 \pm 5.2$ \\
\hline Total & $10.9 \pm 8.1$ & $10.4 \pm 6.5$ \\
\hline & \multicolumn{2}{|c|}{ Mean } \\
\hline \multicolumn{3}{|l|}{ Drug expenditure/episode (\$) } \\
\hline Primary empiric therapy & 4144 & 1593 \\
\hline $\begin{array}{l}\text { Alternative or additional } \\
\text { antifungal therapy }\end{array}$ & 2235 & 3624 \\
\hline Total & 6379 & 5217 \\
\hline
\end{tabular}

episodes and seven (22\%) voriconazole episodes. In the L-AmB group, rash, diarrhea, and nausea were reported. Voriconazole-treated patients experienced rash (four episodes), diarrhea (two episodes), or central nervous system disturbances (one episode), which included confusion, hallucinations other than visual hallucinations, and instability. Rash secondary to voriconazole therapy was most often described as diffuse, erythematous, and maculopapular.

Numbers of episodes in which empiric antifungal therapy was discontinued because of adverse effects did not significantly differ.

\section{Composite Outcome}

No significant difference was noted between the L-AmB and voriconazole groups in the number of episodes in which the composite outcome was achieved (Table 2).

\section{Health Care Resource Utilization}

The mean duration of hospital stay was $32.0 \pm$ 13.9 days for L-AmB-treated patients compared with $29.6 \pm 12.4$ days for voriconazole-treated patients $(p=0.313)$. Treatment durations and costs are compared in Table 4.

Mean drug expenditures/episode for the empiric agent alone were substantially lower in the voriconazole group than in the L-AmB group (\$1593 vs \$4144). Adjusted for the mean duration of therapy, these costs were $\$ 153$ and $\$ 380 /$ day for voriconazole and L-AmB, respectively. Mean costs of antifungal therapy (not including the initial antifungal agent) used in lieu of or in addition to the initial agent were higher with voriconazole than with L-AmB (Table 4); this result was consistent with the finding that a switch to or the addition of an alternative antifungal agent occurred most frequently in the voriconazole group. Despite the high cost of using alternative or additional antifungal drugs, overall mean drug expenditures/episode after we accounted for all antifungals used were still lower for voriconazoletreated patients than for L-AmB-treated patients (Table 4). Antifungal spending accounted for $27 \%$ and $37 \%$ of total pharmacy costs in the voriconazole and L-AmB groups, respectively. Total hospital costs/episode were similar for voriconazole versus L-AmB (\$56,621 vs $\$ 56,495)$.

Comparison of Use of Empiric Antifungal Drugs Before and After Algorithm Implementation

Figures 2 and 3 illustrate the use of L-AmB before implementation and voriconazole after implementation of the algorithm, respectively.

In $81 \%$ of the episodes with L-AmB occurring before implementation of the febrile neutropenia treatment algorithm, therapy was continued without interruption. In $15 \%$ of episodes, L-AmB was discontinued, and voriconazole was started because of adverse effects (two episodes), possible breakthrough invasive fungal infection (one episode), or a desire to switch to oral antifungal therapy (one episode). In one L-AmB episode, combination antifungal therapy with voriconazole was used to treat a possible breakthrough invasive fungal infection.

After the febrile neutropenia treatment algorithm was implemented, L-AmB was used in five episodes. In four of these episodes, L-AmB was used in accordance with the algorithm in that patients were not eligible to receive voriconazole because of elevated hepatic enzyme 
concentrations (three episodes) or therapy with gemtuzumab (one episode).

In $53 \%$ of voriconazole episodes, therapy was continued without interruption. In 34\% of episodes, voriconazole was discontinued and LAmB was started. Primary reasons for these changes were a perceived lack of efficacy in seven cases, followed by adverse effects in two and breakthrough invasive fungal infection in two. In four (13\%) episodes, combination antifungal therapy with L-AmB (three episodes) or caspofungin (one episode) was used. In two cases, combination therapy was used to treat a possible breakthrough invasive fungal infection.

\section{Discussion}

Invasive fungal infections are among the leading causes of death in patients with neutropenic cancer and pose diagnostic and therapeutic challenges. Empiric antifungal therapy has become the standard of care for patients with neutropenia and fever that persists despite broad-spectrum antibacterial therapy., 14-16

Although empiric antifungal therapy is now widely accepted, the antifungal of choice remains controversial. ${ }^{17-22}$ Results of two small studies helped to establish amphotericin B deoxycholate as the standard against which all other antifungal agents would later be compared. ${ }^{23,24}$ Since then, several agents have been evaluated for their comparative efficacy in the empiric treatment of fungal infections during febrile neutropenia. ${ }^{2,4}$,

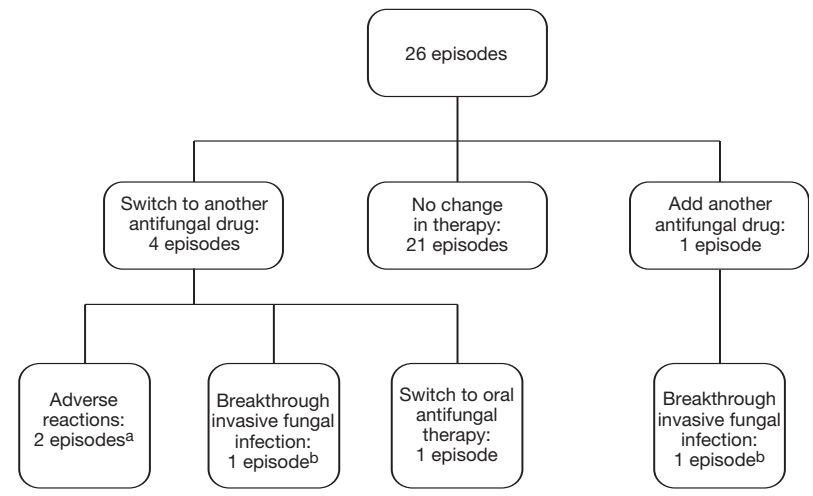

Figure 2. Empiric use of liposomal amphotericin B (L$A m B$ ) before implementation of the febrile neutropenia treatment algorithm. aA third episode in which L-AmB was discontinued because of an adverse reaction was reported, but it did not lead to a switch to another antifungal agent. ${ }^{b}$ This was one of three (all classified as possible) breakthrough invasive fungal infections reported in the LAmB group; therapy with L-AmB was continued in the remaining episode.
25-32 The United States Food and Drug Administration has approved four agents-amphotericin B deoxycholate, L-AmB, itraconazole, and caspofungin-for this indication. ${ }^{33-36}$

Although voriconazole was evaluated in one of the largest studies of febrile neutropenia, its relative efficacy compared with that of L-AmB remains highly debated. The only randomized controlled study to compare voriconazole with L$A m B$ in the setting of neutropenic fever did not demonstrate that voriconazole was noninferior to L-AmB. ${ }^{4}$ No significant difference was observed in the overall response rate (as evaluated by using a composite outcome) between L-AmBand voriconazole-treated patients $(30.6 \%$ vs $26.0 \%, 95 \%$ confidence interval for the difference -10.6-1.6). However, post hoc analyses of the individual outcome variables demonstrated a significantly lower occurrence of breakthrough invasive fungal infections with voriconazole than with L-AmB (8 vs 21, p=0.02). This finding was controversial. ${ }^{18,37}$ However, coupled with the established effectiveness of voriconazole for treating invasive fungal infections, the finding stimulated interest in voriconazole as a feasible alternative to L-AmB. ${ }^{10,38}$

Despite the few episodes involved in our retrospective analysis, we observed statistically and clinically significant differences in relevant effectiveness, safety, and economic outcomes for voriconazole compared with $\mathrm{L}-\mathrm{AmB}$ in a clinical practice setting. Whether implementation of the febrile neutropenia treatment algorithm alone influenced these outcomes cannot be entirely excluded. ${ }^{39}$ Rates of breakthrough invasive fungal infections at our institution were similar

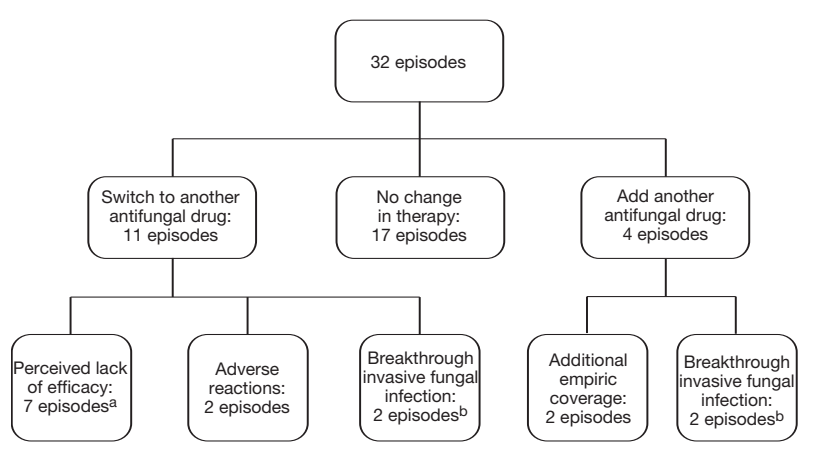

Figure 3. Empiric use of voriconazole after implementation of the febrile neutropenia treatment algorithm. aSeven of eight episodes in which the drug was discontinued because of a perceived lack of efficacy. ${ }^{b}$ These were two of four (three possible, one proven) breakthrough invasive fungal infections reported in the voriconazole group. 
to those reported in the literature. An association between prophylactic use of voriconazole and an increased frequency of zygomycosis has been suggested. ${ }^{40}$ This suggestion is notable in so far as the only proven breakthrough invasive fungal infection in our voriconazole group was due to Mucor species. However, whether this event was related in any way to the exposure to voriconazole is unknown.

The rate of premature discontinuation due to a perceived lack of efficacy was highest in the voriconazole group. This finding may reflect lack of prescriber confidence in voriconazole given the limited clinical experience with this agent when the new algorithm was being implemented. The relatively frequent switch to or the addition of an alternative antifungal agent in the voriconazole group increased expenditures on other antifungals in voriconazole-treated patients (\$3624) compared with L-AmB-treated patients (\$2235). In the converse, the lack of alternative antifungals, such as voriconazole and caspofungin, for empiric therapy before implementation of the algorithm in January 2003 may have contributed to the low rates of discontinuation in the L-AmB group. In addition, our algorithm recommended a switch to L-AmB after only 72 hours of treatment with voriconazole for patients who had persistent fever. This period may have been too short to enable the clinician to assess antifungal effectiveness, and it may have contributed to the relatively high rates of premature discontinuation in the voriconazole group.

Consistent with the safety profile of the agents, infusion-related reactions, elevated serum creatinine concentrations, and electrolyte disturbances were most common in the L-AmB group. Although elevated hepatic enzyme levels and visual disturbances were reported in the voriconazole group, they occurred in few patients. Visual disturbances contributed to the discontinuation of voriconazole in two episodes. The adverse effects that led to one drug discontinuation in the L-AmB group were serious infusion-related reactions.

Based on these results, we continue to use this algorithm at our institution for the empiric treatment of fungal infections in adult patients with hematologic cancer and febrile neutropenia. However, the use of voriconazole as the primary empiric antifungal agent is not without its limitations, which include the potential for serious cytochrome P450-based drug-drug interactions and the development of infections from Zygomycetes species. Voriconazole lacks activity against these pathogens, ${ }^{40}$ which are associated with a high mortality rate in immunocompromised patients with prolonged neutropenia.

The noninferiority of caspofungin to L-AmB was demonstrated in a clinical trial similar to that conducted to compare L-AmB and voriconazole. ${ }^{32}$ However, caspofungin has not replaced voriconazole in the febrile neutropenia treatment algorithm at this time because it does not confer advantages over voriconazole with regard to ease of administration or institutional acquisition cost. In addition, outcomes data suggesting the superiority of either agent from a safety or effectiveness perspective in the setting of febrile neutropenia are not yet available.

\section{Study Limitations}

The retrospective and observational nature of this study limited our ability to control for bias; however, the similarities between the L-AmB and voriconazole groups in demographic and clinical characteristics suggest that the groups were well balanced with respect to their risks of acquiring invasive fungal infections. ${ }^{1,41}$ Furthermore, an internal registry of all chemotherapy regimens that patients with acute leukemia received at our institution suggests that treatment outcomes directly attributable to these regimens have remained relatively unchanged over the past 4 years. Antifungal prophylaxis or modifications to antibacterial therapy made immediately before the start of empiric antifungal therapy likely had minimal confounding effects given that these events occurred in similar numbers of L-AmB and voriconazole episodes.

Although we based our outcome measures on well-established definitions of efficacy and safety used in previous studies of empiric antifungal therapy, assessment of these outcomes is particularly challenging in a retrospective study. ${ }^{42}$ Given the rarity of breakthrough invasive fungal infections, an unrealistically large number of patients are often required to achieve adequate statistical power to demonstrate significant differences between agents with regard to this outcome. Also, in the retrospective setting, confirmation of a breakthrough invasive fungal infection depended on the availability of culture results, which may not have been obtained before we evaluated for the presence of infection (i.e., 7 days after discontinuation of the empiric antifungal agent). Use of defervescence as an outcome measure is also limited because of its 
lack of specificity for occult fungal infection, and it can be highly variable given the arbitrary nature of when it is assessed relative to the administration of antifungal therapy.

We attempted to address some of these limitations by incorporating a composite outcome into our effectiveness measures and by not relying solely on one end point to define clinical effectiveness. Furthermore, despite the small sample size, statistically significant differences between the L-AmB and voriconazole groups were observed for certain effectiveness and safety outcomes. A study larger than ours is unlikely to reveal statistically significant differences between these agents given the similar responses seen in the L-AmB and voriconazole groups, especially with respect to defervescence and breakthrough invasive fungal infections. We chose not to report any post hoc power calculations to determine if our study had adequate power to reveal differences between the groups. The reason was because this practice has widely been discouraged as a means of explaining nonsignificant results. ${ }^{43,}{ }^{44}$ Differences between antifungal agents in the setting of febrile neutropenia may not be evident if patients have a low risk of acquiring an invasive fungal infection at baseline; the results may reflect only the futility of both agents. ${ }^{42}$ However, the characteristics of our population (i.e., patients with hematologic malignancies and prolonged neutropenic fever who were primarily undergoing highly immunosuppressive induction chemotherapy) suggest that this was not the case in our study. Ours was the subset of patients considered to be at highest risk for invasive fungal infection. ${ }^{1,41}$

In addition, clinicians should exercise caution when extrapolating our results to different institutions and patient populations (i.e., recipients of hematopoietic stem cell transplants). These results are specific to our patient population and may vary given differences among institutions in terms of patients' clinical and demographic characteristics, chemotherapy and study protocols, patterns of antibiotic use, strategies for antifungal prophylaxis, and commonly encountered pathogens.

\section{Conclusion}

This retrospective analysis examined our institution's febrile neutropenia treatment algorithm that incorporates voriconazole as the primary empiric antifungal agent to treat febrile neutropenia in adults; the analysis demonstrated that voriconazole had effectiveness outcomes comparable to those of L-AmB, and was associated with lower costs and fewer adverse effects.

\section{Acknowledgment}

We would like to thank Bradley J. McCloskey, Pharm.D., for his assistance with data collection.

\section{References}

1. Hughes WT, Armstrong D, Bodey GP, et al, for the Infectious Diseases Society of America. 2002 guidelines for the use of antimicrobial agents in neutropenic patients with cancer. Clin Infect Dis 2002;34:730-51.

2. Walsh TJ, Finberg RW, Arndt C, et al, for the National Institute of Allergy and Infectious Diseases Mycoses Study Group. Liposomal amphotericin B for empirical therapy in patients with persistent fever and neutropenia. N Engl J Med 1999;340:764-71.

3. Wingard JR, White M, Anaissie E, Raffalli J, Goodman J, Arrieta A, for the L Amph/ABLC Collaborative Study Group. A randomized, double-blind comparative trial evaluating the safety of liposomal amphotericin B versus amphotericin B lipid complex in the empirical treatment of febrile neutropenia. Clin Infect Dis 2000;31:1155-63.

4. Walsh TJ, Pappas P, Winston DJ, et al, for the National Institute of Allergy and Infectious Diseases Mycoses Study Group. Voriconazole compared with liposomal amphotericin B for empirical antifungal therapy in patients with neutropenia and persistent fever. N Engl J Med 2002;346:225-34.

5. Espinel-Ingroff A. In vitro fungicidal activities of voriconazole, itraconazole, and amphotericin B against opportunistic moniliaceous and dematiaceous fungi. J Clin Microbiol 2001;39:954-8.

6. Kappe R. Antifungal activity of the new azole UK-109, 496 (voriconazole). Mycoses 1999;42(suppl 2):83-6.

7. Hoban DJ, Zhanel GG, Karlowsky JA. In vitro susceptibilities of Candida and Cryptococcus neoformans isolates from blood cultures of neutropenic patients. Antimicrob Agents Chemother 1999;43:1463-4.

8. Ally R, Schurmann D, Kreisel W, et al, for the Esophageal Candidiasis Study Group. A randomized, double-blind, double-dummy, multicenter trial of voriconazole and fluconazole in the treatment of esophageal candidiasis in immunocompromised patients. Clin Infect Dis 2001;33: $1447-54$.

9. Denning DW, Ribaud P, Milipied N, et al. Efficacy and safety of voriconazole in the treatment of acute invasive aspergillosis. Clin Infect Dis 2002;34:563-71.

10. Herbrecht R, Denning DW, Patterson TF, et al, for the Invasive Fungal Infections Group of the European Organisation for Research and Treatment of Cancer and the Global Aspergillus Study Group. Voriconazole versus amphotericin B for primary therapy of invasive aspergillosis. N Engl J Med 2002;347: 408-15.

11. Pfizer, Inc. Vfend (voriconazole) package insert. New York, NY; 2003.

12. Cohen HE, ed. Drug topics red book. Montvale, NJ: Medical Economics Company, 2003.

13. Ascioglu S, Rex JH, de Pauw B, et al, for the Invasive Fungal Infections Cooperative Group of the European Organization for Research and Treatment of Cancer and Mycoses Study Group of the National Institute of Allergy and Infectious Diseases. Defining opportunistic invasive fungal infections in immunocompromised patients with cancer and hematopoietic stem cell transplants: an international consensus. Clin Infect Dis 2002;34:7-14.

14. Donowitz GR, Maki DG, Crnich CJ, Pappas PG, Rolston KV. 
Infections in the neutropenic patient: new views of an old problem. Hematology (Am Soc Hematol Educ Program) 2001;113-39.

15. Klastersky J. Empirical antifungal therapy. Int J Antimicrob Agents 2004;23:105-12.

16. Martino P, Girmenia C. Making the diagnosis of fungal infection: when to start treatment. Int J Antimicrob Agents 2000;16:323-9.

17. Marr KA. Empirical antifungal therapy: new options, new tradeoffs [editorial]. N Engl J Med 2002;346:278-80.

18. Powers JH, Dixon CA, Goldberger MJ. Voriconazole versus liposomal amphotericin B in patients with neutropenia and persistent fever [comment]. N Engl J Med 2002;346:289-90.

19. Johnson JR. Voriconazole versus liposomal amphotericin B for empirical antifungal therapy [comment]. N Engl J Med 2002;346:1745-7.

20. Elting LS, Cantor SB. Outcomes and costs of febrile neutropenia: adventures in the science and art of treatment choices. Support Care Cancer 2002;10:189-96.

21. Danaher PJ. Caspofungin versus liposomal amphotericin B for empirical therapy [comment]. N Engl J Med 2005;352:410-14.

22. Jorgensen KJ, Gotzsche PC, Johansen HK. Voriconazole versus amphotericin B in cancer patients with neutropenia. Cochrane Database Syst Rev 2006;(1):CD004707.

23. Pizzo PA, Robichaud KJ, Gill FA, Witebsky FG. Empiric antibiotic and antifungal therapy for cancer patients with prolonged fever and granulocytopenia. Am J Med 1982;72: $101-11$

24. EORTC International Antimicrobial Therapy Cooperative Group. Empiric antifungal therapy in febrile granulocytopenic patients. Am J Med 1989;86:668-72.

25. Schneemann M, Imhof A. Caspofungin versus liposomal amphotericin B for empirical therapy [comment]. N Engl J Med 2005;352:410-14

26. Viscoli C, Castagnola E, Van Lint MT, et al. Fluconazole versus amphotericin $B$ as empirical antifungal therapy of unexplained fever in granulocytopenic cancer patients: a pragmatic, multicentre, prospective and randomised clinical trial. Eur J Cancer 1996;32:814-20.

27. Malik IA, Moid I, Aziz Z, Khan S, Suleman M. A randomized comparison of fluconazole with amphotericin B as empiric antifungal agents in cancer patients with prolonged fever and neutropenia. Am J Med 1998;105:478-83.

28. Winston DJ, Hathorn JW, Schuster MG, Schiller GJ, Territo MC. A multicenter, randomized trial of fluconazole versus amphotericin B for empiric antifungal therapy of febrile neutropenic patients with cancer. Am J Med 2000;108:282-9.

29. White MH, Bowden RA, Sandler ES, et al. Randomized, double-blind clinical trial of amphotericin B colloidal dispersion vs. amphotericin B in the empirical treatment of fever and neutropenia. Clin Infect Dis 1998;27:296-302.

30. Cagnoni PJ, Walsh TJ, Prendergast MM, et al. Pharmacoeconomic analysis of liposomal amphotericin B versus conventional amphotericin B in the empirical treatment of persistently febrile neutropenic patients. J Clin Oncol 2000;18:2476-83.

31. Boogaerts M, Winston DJ, Bow EJ, et al. Intravenous and oral itraconazole versus intravenous amphotericin B deoxycholate as empirical antifungal therapy for persistent fever in neutropenic patients with cancer who are receiving broadspectrum antibacterial therapy: a randomized, controlled trial. Ann Intern Med 2001;135:412-22.

32. Walsh TJ, Teppler H, Donowitz GR, et al. Caspofungin versus liposomal amphotericin B for empirical antifungal therapy in patients with persistent fever and neutropenia. N Engl J Med 2004:351:1391-402.

33. X-GEN Pharmaceuticals Inc. Amphotericin B for injection package insert. Northport, NY; 2003.

34. Fujisawa Healthcare, Inc. AmBisome (amphotericin B) liposome for injection package insert. Deerfield, IL; 2002.

35. Ortho Biotech. Sporanox (itraconazole) injection package insert. North Chicago, IL; 2004

36. Merck \& Co, Inc. Cancidas (caspofungin acetate) for injection package insert. Whitehouse Station, NJ; 2005.

37. Powers JH. Empirical antifungal therapy in febrile neutropenic patients: caution about composite end points and the perils of $\mathrm{P}$ values [comment]. Clin Infect Dis 2004;39:1738-9.

38. Kullberg BJ, Sobel JD, Ruhnke M, et al. Voriconazole versus a regimen of amphotericin $\mathrm{B}$ followed by fluconazole for candidaemia in non-neutropenic patients: a randomised noninferiority trial. Lancet 2005;366:1435-42.

39. Grimshaw JM, Russell JT. Effect of clinical guidelines on medical practice: a systematic review of rigorous evaluations. Lancet 1993;342:1317-22.

40. Kauffman CA. Zygomycosis: reemergence of an old pathogen. Clin Infect Dis 2004;39:588-90.

41. Klastersky J, Paesmans M, Rubenstein EB, et al. The multinational association for supportive care in cancer risk index: a multinational scoring system for identifying low-risk febrile neutropenic cancer patients. J Clin Oncol 2000;18: 3038-51.

42. Bennett JE, Powers J, Walsh T, et al. Forum report: issues in clinical trials of empirical antifungal therapy in treating febrile neutropenic patients. Clin Infect Dis 2003;15(36 suppl 3):S117-22.

43. Hoenig JM, Heisery DM. The abuse of power: the pervasive fallacy of power calculations for data analysis. Am Stat 2001;55:19-24.

44. Levine M, Ensom MHH. Post hoc power analysis: an idea whose time has passed? Pharmacotherapy 2001;21:405-9. 